CERN-EP/99-99

15 July 1999

\title{
RESOLUTION LIMITS OF DRIFT TUBES
}

\author{
W. Riegler ${ }^{a, 1}$, M. Aleksa ${ }^{a}$, M. Deile ${ }^{b}$, J. Dubbert ${ }^{b}$, C.W. Fabjan ${ }^{a}$, \\ C. Gruhn ${ }^{c}$, N.P. Hessey ${ }^{b, 2}$, T. Sammer ${ }^{b, 3}$
}

\begin{abstract}
Measurements of the drift-tube response to charged particle tracks are compared with a complete simulation. The measured resolution of typically $80 \mu \mathrm{m}$ agrees well with the simulation and allows the individual factors limiting the resolution such as diffusion, charge deposit fluctuations, gas gain fluctuations and signal processing to be studied. The results with respect to the dependence of the drift chamber resolution on gas gain, gas pressure and electronics parameters are reported.
\end{abstract}

${ }^{a}$ EP Division, CERN, CH-1211 Geneva 23, Switzerland.

${ }^{b}$ Ludwig-Maximilians-Universität München, 80539 München, Germany.

${ }^{c}$ Max-Planck-Institut für Physik, Föhringer Ring 6, 80805 München, Germany.

${ }^{1}$ Now at Harvard University, 42 Oxford Street, Cambridge MA 02138, USA.

${ }^{2}$ Now at NIKHEF, Kruislaan 409, 1098 SJ Amsterdam, Netherlands.

${ }^{3}$ Now at Max-Planck-Institut für Physik, Föhringer Ring 6, 80805 München, Germany. 


\section{Introduction}

Cylindrical drift tubes will be the precision tracking devices for the muon spectrometer of ATLAS [?, ?], a general purpose experiment at the Large Hadron Collider (LHC) at CERN. About 370000 drift tubes will be assembled into 1200 chambers to measure the muon tracks along a spectrometer arm of $5-15 \mathrm{~m}$. To achieve a momentum resolution of $10 \%$ for a $1 \mathrm{TeV}$ muon, the position resolution of each measurement must be better than $80 \mu \mathrm{m}$. Although single-wire space resolutions of better than $20 \mu \mathrm{m}$ have been achieved in drift chambers, the goal of $80 \mu \mathrm{m}$ is a new challenge due to the large size of the system, the high background counting rates of up to $300 \mathrm{kHz} /$ wire, and economic constraints.

The most important requirements for a drift chamber in this high-rate environment are, in principle, a relatively fast drift velocity to reduce the occupancy, low gas gain to avoid chamber ageing effects due to large amounts of charge deposit on the wire, and linearity of the space-drift-time relation to minimize space-charge effects. However, since a good position resolution would favour high gas gain and a slow drift gas, a detailed study was performed of all the resolution limiting parameters in order to achieve good resolution in this very 'hostile' environment even for low gain and fast gases. The drift chamber resolution was measured and compared with a full chain simulation of the detector. The good agreement allowed us to make a detailed study of the individual components that contribute to the resolution and also showed that we have a satisfactory understanding of the drift chamber processes. Techniques to improve the resolution are also presented.

\section{Chamber geometry and measurement set-up}

The drift tube working point parameters are listed in Table 1, and the experimental set-up is shown in Fig. ??. The tube radius is $1.5 \mathrm{~cm}$, the gas pressure is 3 bars.

Table 1

Drift tube parameters.

\begin{tabular}{|l|l|}
\hline Inner/Outer tube radius & $1.46 / 1.5 \mathrm{~cm}$ \\
Tube length & $1 \mathrm{~m}$ \\
Wire diameter & $50 \mu \mathrm{m} \mathrm{W} / \mathrm{Re} 93 / 7$ \\
Gas & $\mathrm{Ar} / \mathrm{N}_{2} / \mathrm{CH}_{4} 91 / 4 / 5$ at 3 bars absolute [?] \\
Gas gain & $2 \times 10^{4}(3270 \mathrm{~V})$ \\
Maximum drift time & $480 \mathrm{~ns}$ \\
Preamp peaking time & $15 \mathrm{~ns}$ \\
Discriminator threshold & 18 primary electrons (see text) \\
\hline
\end{tabular}

Five drift tubes were operated in a $170 \mathrm{GeV}$ muon beam at CERN. One end was read out with a current-sensitive preamplifier [?], the other end was terminated at the characteristic tube impedance of $380 \Omega$ in order to avoid signal reflections. The amplifier output is driven to a discriminator and a TDC with a timing resolution of 300 ps r.m.s. Two scintillators, read out on both sides, trigger the experiment with a timing resolution of $<0.5 \mathrm{~ns}$. 


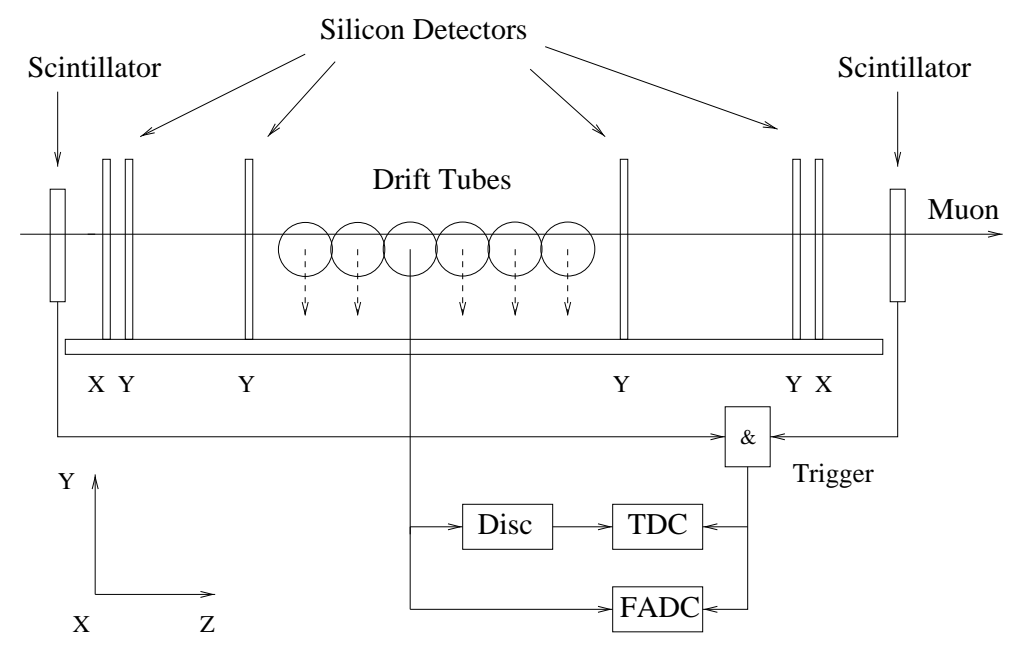

Figure 1: Measurement set-up.

The discriminator threshold is set to five times the noise r.m.s. voltage which is dominated by the thermal noise of the termination resistor [?][?]. To determine the drift tube resolution, a silicon strip detector measured the position of the muon track at the tube position with an accuracy of $<10 \mu \mathrm{m}$.

We will refer to the threshold in units of primary electrons (p.e.) defined as the peak of a pulse created by a single ionization electron. Experimentally, this number is found by measuring the pulse height caused by ${ }^{55} \mathrm{Fe}$ photons $(5.9 \mathrm{keV})$ that produce a very localized cluster of 226 electrons in the detector (the effective ionization energy of argon is $26 \mathrm{eV}$ ). The $5 \times$ r.m.s. noise level was measured to correspond to a level of 18 p.e. for a gas gain of $2 \times 10^{4}$.

It is very useful to express the threshold in these units which indicates the number of electrons required to arrive at the wire within the integration time of the electronics such that the output pulse crosses the discriminator threshold.

\section{$3 \quad$ Experimental results}

The TDC data plotted against the distance of the muon track from the wire are shown in Fig. ??. The width of the band corresponds to the MDT resolution, the events below the band correspond to electromagnetic secondaries (e.g. delta rays) produced by the muon which obscure the actual muon track. The TDC data were averaged in $1 \mathrm{~mm}$ slices and fitted with a Gaussian to extract the space-drift-time relation.

Finally, the drift chamber resolution was calculated by comparing the track position predicted by the silicon telescope and the track position calculated from the TDC data using the space-drift-time relation, and fitting the residual distribution with a Gaussian. The drift chamber resolution as a function of the distance from the wire together with the results of a detailed simulation can be seen in Fig. ??. 


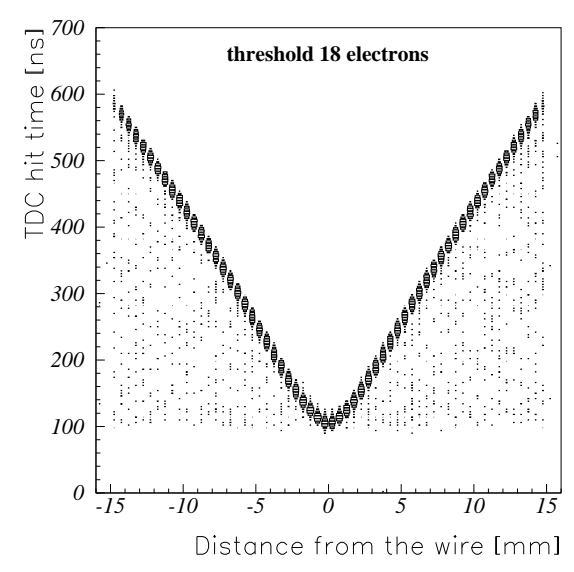

Figure 2: TDC times versus distance of the muon track from the wire. The data are collected in radial bins of $0.5 \mathrm{~mm}$.

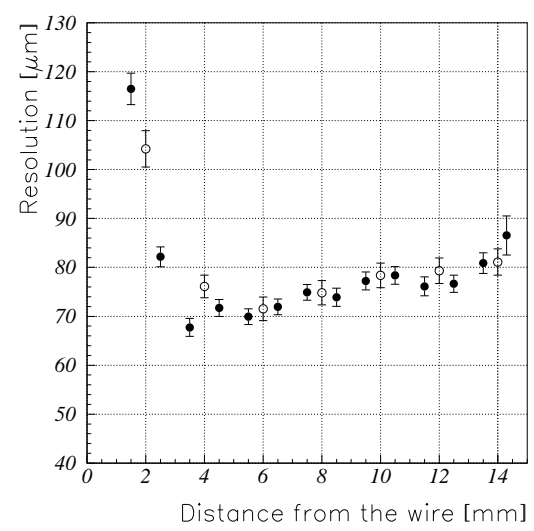

Figure 3: Resolution versus drift distance. The solid points indicate the measurements, the open points show the simulations.

\section{$4 \quad$ Drift tube simulation}

The drift tube response to charged particle tracks was simulated using a drift chamber simulation program called GARFIELD [?], a program for calculating the transport properties of gas mixtures called MAGBOLTZ [?], and a program for calculating the energy deposit of fast particles in gases called HEED [?]. MAGBOLTZ and HEED are interfaced to GARFIELD. The preamp delta response was measured on the set-up by injecting a current delta pulse (voltage step through a $1 \mathrm{pf}$ capacitor) into the tube. The peaking time was $15 \mathrm{~ns}$, a number that will be justified later.

A single event was simulated in the following way: HEED calculates the ionization along the particle track, and GARFIELD tracks the individual ionization electrons in the electric field according to the transport properties, i.e. the diffusion and drift velocity as calculated by MAGBOLTZ. The average number of ionization electrons is about 300 electrons $/ \mathrm{cm}$. The induced current signal is calculated by tracking the ions, produced in the avalanche, to the cathode. The drift time of the ions to the cathode is $3.8 \mathrm{~ms}$. 
After adding electronics noise to the signal it is convoluted with the preamplifier delta response. Finally, the threshold crossing time of the signal is found.

The electronics noise, which imposes a lower limit on the threshold setting, is dominated by the thermal noise of the termination resistor. The thermal Equivalent Noise Charge (ENC) from the termination resistor is given by

$$
E N C=\sqrt{\frac{k T}{R_{t}} \tau}=0.4 \mathrm{fC},
$$

where $k$ is the Boltzmann constant, $\tau=15 \mathrm{~ns}$ the preamplifier peaking time, $R_{t}=380 \Omega$ the termination resistor value, and $T=300 \mathrm{~K}$ the resistor temperature. Dividing the $5 \times$ noise amplitude r.m.s. by the peak of a simulated single electron pulse gives a value of 16 p.e. which is very close to the measurement (18 p.e.) and indicates that the drift tube was operated close to the theoretical noise level. The only parameters that have to be taken from measurements are the gas gain for a specific voltage and the ion mobility. In principle, MAGBOLTZ is able to calculate the gas gain, but as the avalanche multiplication is an exponential process, the errors are large. It is also important to note that the electric field near the wire is very high $\left(2.2 \times 10^{5} \mathrm{~V} / \mathrm{cm}\right)$, so the field dependence of the ion mobility starts to be significant [?].

A sample of 5000 tracks at random distances was created as described above and analysed in the same way as the actual data. A comparison of the measurements with the simulation is shown in Fig. ??. It is important to notice that there are no free parameters in this simulation: the good agreement with the measurements demonstrates that we have a complete understanding of all the individual processes which allows us to study the individual contributions limiting the resolution.

\section{$5 \quad$ Resolution-limiting contributions}

Knowing that the simulation reproduces the measurements well, we can identify individual resolution limiting contributions by 'switching them off' in simulation and looking at the resolution change. The dominant resolution-limiting parameters are cluster position fluctuations, ionization fluctuations, diffusion, gas gain fluctuations, and electronics noise, which we discuss in detail:

- Cluster position fluctuations, i.e. the non-uniform separation of the primary ionization electrons along the muon track, result in different arrival times of the first electrons due to different drift distances to the anode wire. Since the primary interactions are independent, the distance between ionization clusters is distributed exponentially. This contribution is small because the ionization density is very large, due to the high gas pressure, as can be seen in Fig. ??. The contribution decreases with increasing distance from the wire because a given separation of two clusters translates into a smaller arrival time difference.

- Cluster ionization fluctuations, i.e. the fluctuations of the actual amount of charge deposited by the muon, are significant (Fig. ??). The finite rise time of the preamplifier (15 ns) causes different threshold crossing times for different charge deposits, and hence reduced resolution (time slewing). The time-slewing effect has a significant impact on the resolution close to the wire: the threshold crossing time fluctuations 
caused by the different signal rise times translate into large position errors due to the high drift velocity close to the wire.

- The effect of diffusion increases with the distance from the wire as one would expect (Fig. ??).

- Electronics noise has a small direct impact compared to diffusion and charge fluctuations (Fig. ??). However, it indirectly affects the resolution dramatically since it entails a lower limit for the threshold. If the noise were to be smaller (or the gas gain higher) one could decrease the threshold and reduce the time-slewing effect.

- Gas gain fluctuations have a very small impact since the primary ionization fluctuations are far bigger.

It should be noted that the individual resolution limiting contributions are not at all independent. As an example, the diffusion effect is strongly correlated with charge fluctuations, i.e. with a large charge deposit the signal rises faster, which shifts the threshold crossing to earlier times (time slewing). However, there are also more electrons that experience diffusion as they drift to the wire. The first electrons causing the threshold crossing are further away from the centre of gravity of the electron cloud which also shifts the threshold crossing to earlier times. It was found that the square sum of the two individual effects results in a resolution which is almost $30 \%$ lower compared to the combined effect. Electronics noise is correlated with the charge fluctuations in exactly the opposite way. With small charge deposits (slow rise time), the noise shifts the threshold crossing time, on average, to an earlier time compared to a fast rise time signal, so the square sum of the noise and charge fluctuation contribution is worse than the combined effect.

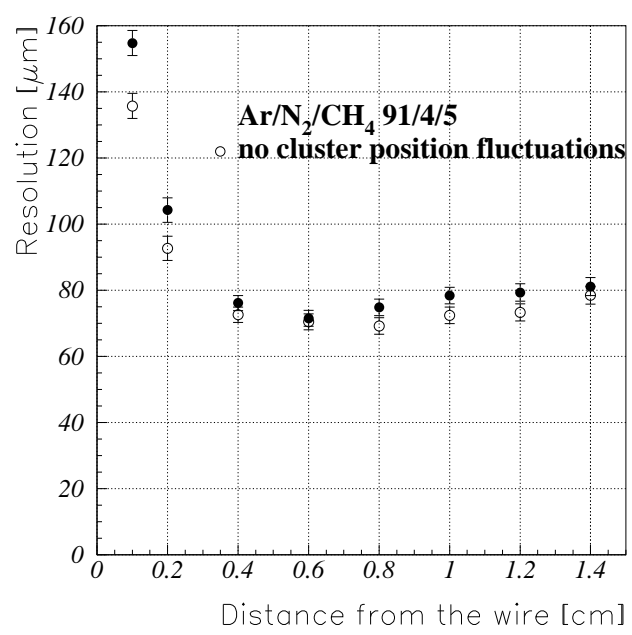

Figure 4: The open circles show the simulated resolution assuming a uniform distribution of the ionization clusters. The non-uniform cluster distribution has only a small impact on the resolution. 


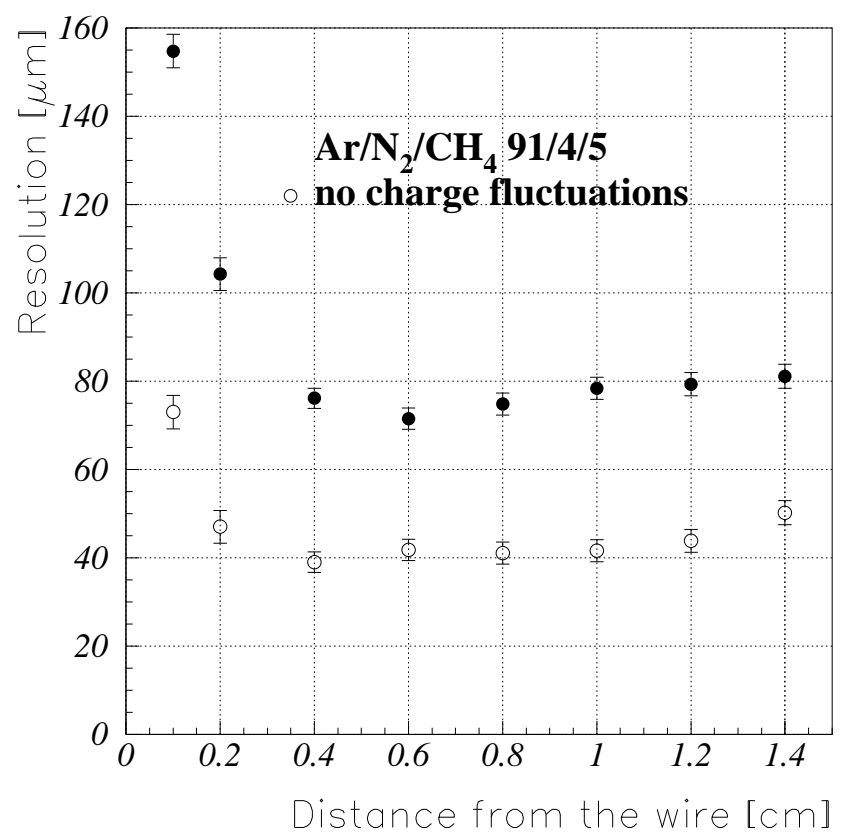

Figure 5: The open circles show the simulated resolution assuming a constant amount of charge (equal to the average charge deposit) in each ionization cluster. The charge deposit fluctuations have a significant impact on the resolution because of time-slewing effects.

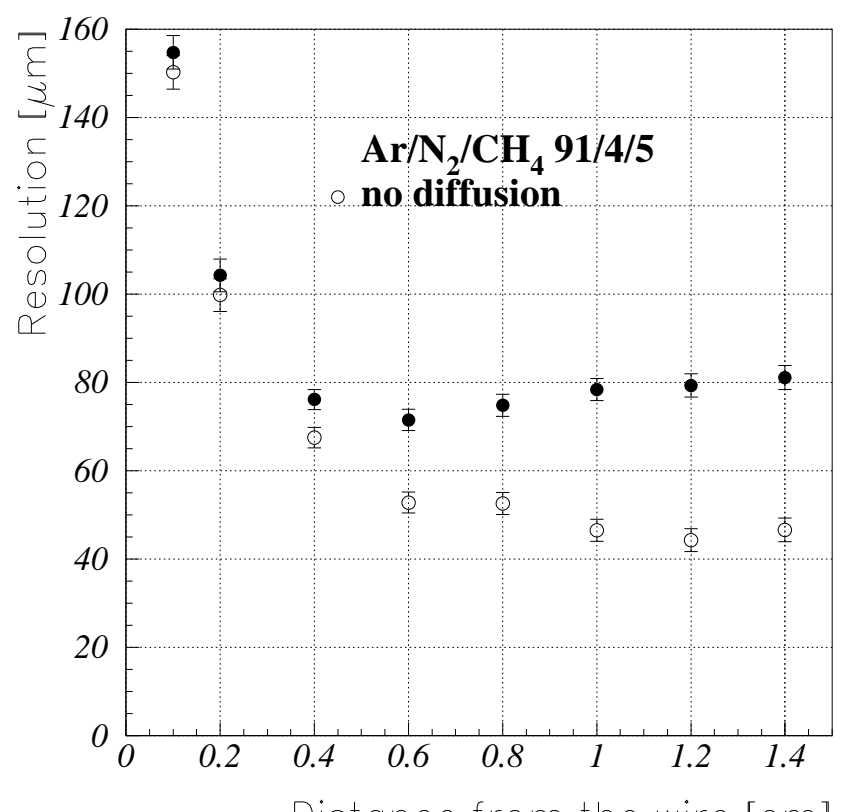

Figure 6: The open circles show the simulated resolution neglecting the diffusion effect. This contribution increases with the distance of the track from the wire. 


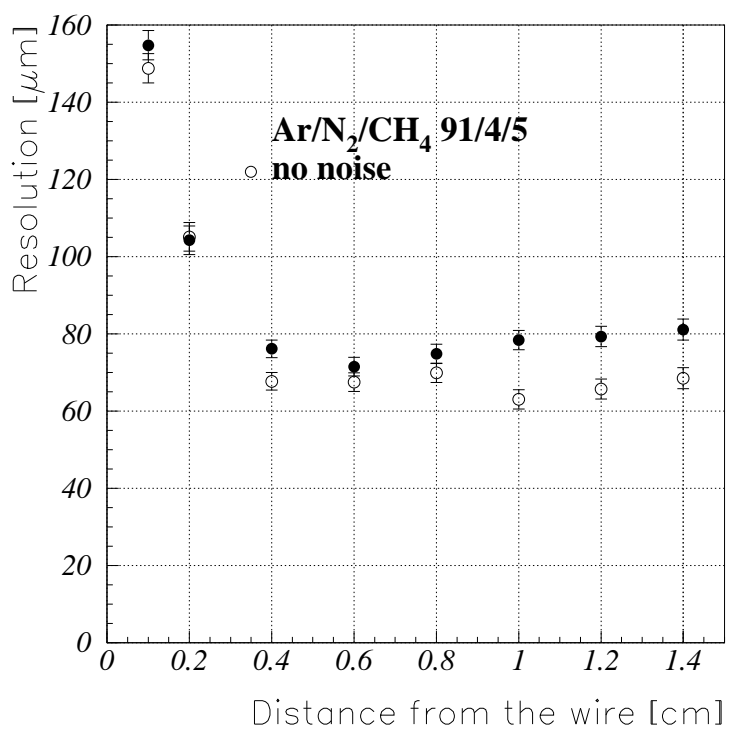

Figure 7: The open circles show the simulated resolution neglecting the noise. The direct contribution is small.

\section{Resolution optimization}

Based on this quantitative understanding of the individual contributions we can aim to improve performance. Diffusion can be reduced by increasing the gas pressure (or using a gas with a lower diffusion coefficient). Since the total amount of charge deposit is the main limitation imposed by tube ageing, this comparison is made for the same amount of charge deposited on the anode wire; operating at a pressure of one bar would give us a three times higher gas gain compared to 3 bars (Fig. ??). One notices a dramatic improvement from one to three bars but only a marginal improvement for higher pressures because the other contributions start to dominate the resolution.

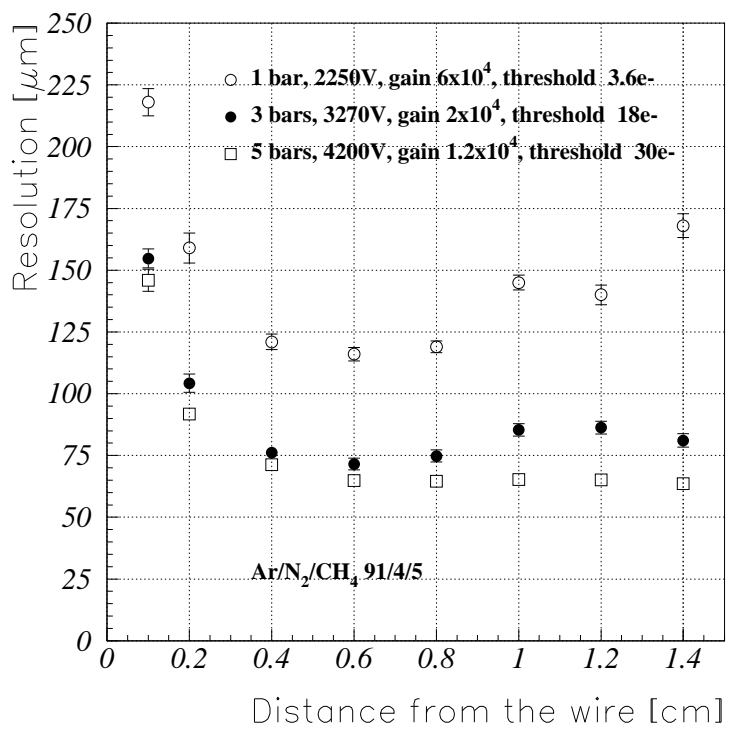

Figure 8: Simulated resolution for different gas pressures for the same amount of charge deposit. The resolution improves with pressure due to reduced diffusion and reduced charge fluctuations. 
There are several ways to reduce the contribution from charge fluctuations: a gas mixture with a slower drift velocity will reduce this effect since the same threshold crossing time fluctuations will translate (via the space-drift-time relation) into smaller fluctuations of the reconstructed drift distance. However, in the LHC environment rather short drift times are required in order to reduce the detector occupancy.

Decreasing the threshold reduces the contribution from charge fluctuations, but a lower threshold limit is set by the noise level. The only way to decrease the effective threshold is to increase the gas gain which is, however, limited by the need of a low charge deposit. For ten years of operation in the LHC environment and a gas gain of only $2 \times 10^{4}$ we expect a charge deposit of about $1 \mathrm{C} / \mathrm{cm}$, which we consider an upper limit for the ATLAS drift chambers. The second reason for a low gas gain is the reduction of resolution deteriorating space charge. Figure ?? compares the resolution at a gain of $2 \times 10^{4}$ to a gas gain of $6 \times 10^{4}$, i.e. at an effectively three times lower threshold.

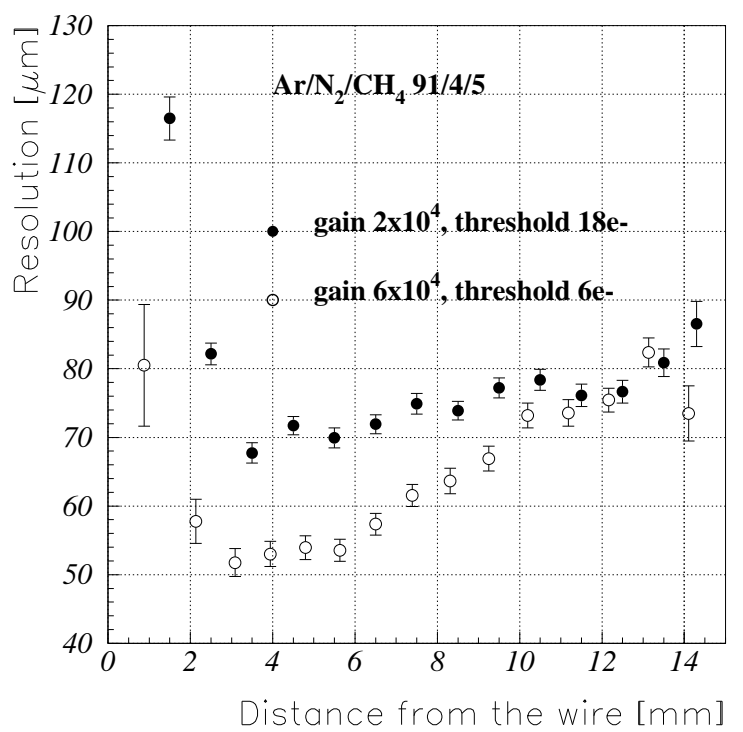

Figure 9: Measured resolution for different gas gains. Increasing the gas gain allows a lower threshold and reduces the time-slewing effect.

Increasing the bandwidth ('speed') of the preamplifier improves the resolution by reducing the time-slewing effect (Fig. ??). The improvement in resolution from $t p=15 \mathrm{~ns}$ to $t p=5 \mathrm{~ns}$ is $10 \mu \mathrm{m}$. This should be compared with the advantages of a slower preamp which is more robust against pickup noise and oscillations, a significant advantage in a large system. Another argument in favour of a slower preamp is the number of multiple hits. From Fig. ?? we can infer that a slower preamp gives a much 'smoother' signal which reduces the number of threshold crossings for a single signal. The average number of hits per signal is three for a preamp with a peaking time of $5 \mathrm{~ns}$, and only 1.2 for a peaking time of $15 \mathrm{~ns}$. 


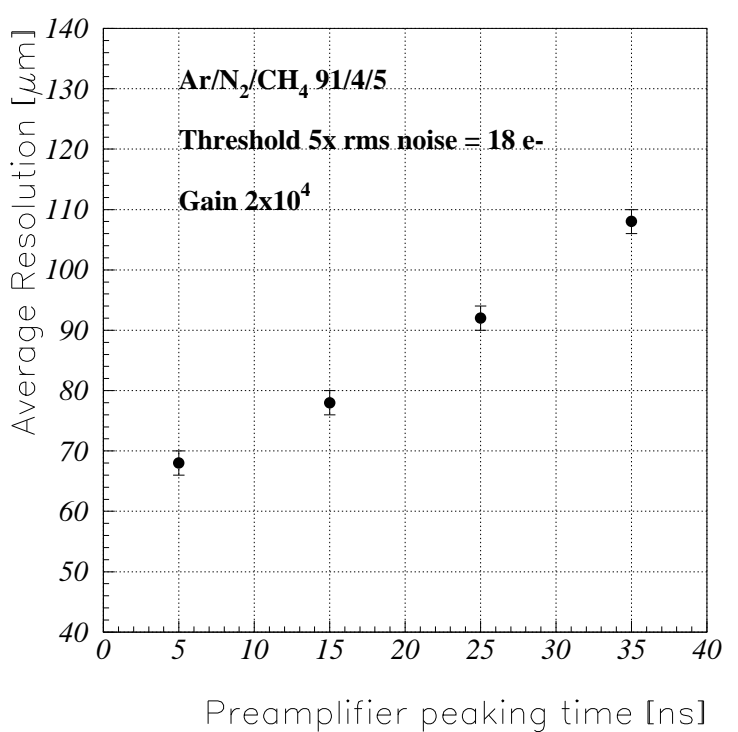

Figure 10: Simulation of the dependence of the resolution on the preamp peaking time. Using fast electronics improves the resolution since it reduces the time-slewing effect.

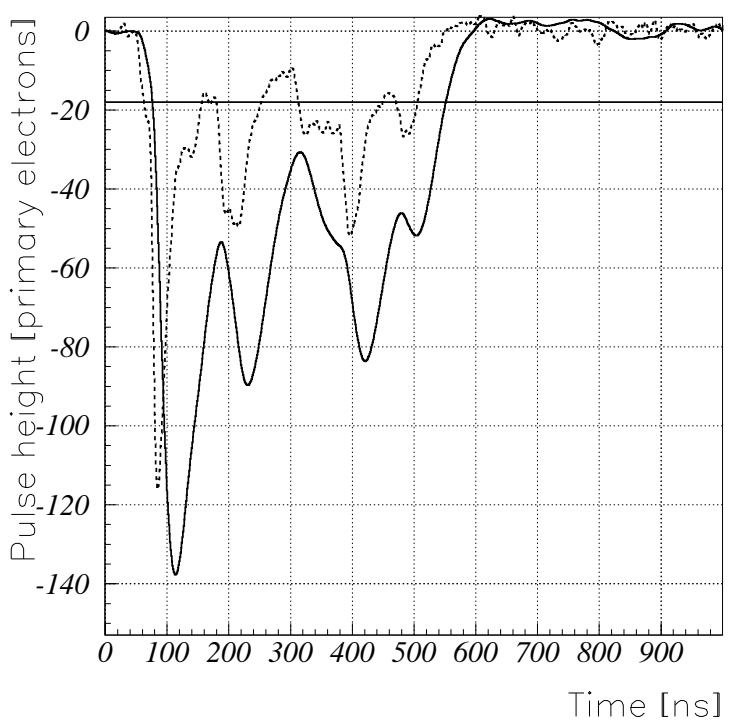

Figure 11: Simulation of the electronics response to the same input pulse for a $t_{p}=5 \mathrm{~ns}$ (dashed line) and a $t_{p}=25 \mathrm{~ns}$ (solid line) preamplifier. The threshold is set to the $5 \times$ r.m.s. noise level (18 p.e.). For short peaking times, a high number of multiple threshold crossings is observed.

Another way to reduce the charge fluctuation contribution is to measure the leading edge charge to correct the threshold crossing time for the time-slewing effect. Figure ?? shows the resolution improvement by correcting the threshold crossing time with the charge measured in a 20 ns gate following the threshold crossing time. The average timeslewing corrected resolution $(64 \mu \mathrm{m})$ is as good as the average resolution for a three times higher gain $(63 \mu \mathrm{m}$, see Fig. ??). The correction compensates for the disadvantage of the low gain and therefore this technique will be adopted in ATLAS. 


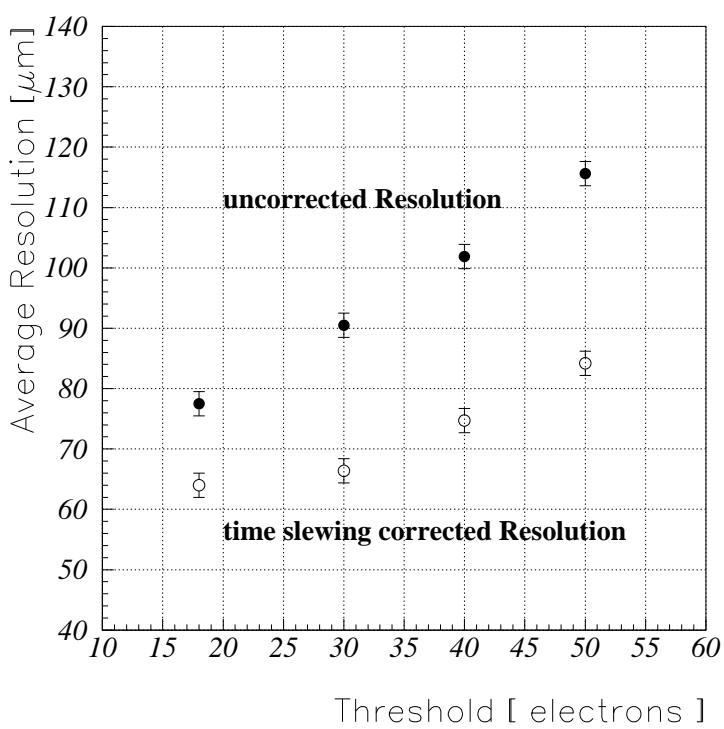

Figure 12: By measuring the leading-edge charge with a short gate ADC (gate $=20 \mathrm{~ns}$ ) one can correct for the time slewing and improve the resolution, as well as reduce the threshold dependence. The figure shows a measurement.

\section{$7 \quad$ Conclusions}

Resolution measurements of cylindrical drift chambers have been performed and compared with a detailed simulation. The individual resolution limiting contributions such as diffusion, charge fluctuations, amplifier peaking time, and electronics noise were studied. The optimized operating parameters for the gas $\mathrm{Ar} / \mathrm{N}_{2} / \mathrm{CH}_{4} 91 / 4 / 5$ are a pressure of 3 bars, a gas gain of $2 \times 10^{4}$, a preamplifier peaking time of $15 \mathrm{~ns}$, and a discriminator threshold of 20 primary electrons $(5 \times$ r.m.s. noise level). The high gas pressure reduces the diffusion contribution. Slow drift velocity, fast preamplifiers, and low thresholds would reduce the contribution from charge fluctuations but require high gas gain and are not compatible with stability and occupancy requirements in high-rate environments. For the above operating parameters a resolution of $80 \mu \mathrm{m}$ was achieved. A time-slewing correction using the signal leading edge charge improves the resolution to $65 \mu \mathrm{m}$ and also reduces the dependence on the threshold. Good agreement is observed between measurements and simulations, indicating that this approach can be extended to other gas mixtures.

\section{References}

[1] ATLAS Technical Proposal, CERN/LHCC/94-43, LHCC/P2 (1994).

[2] Nigel Hessey, The precision drift chambers for the ATLAS Muon Spectrometer, Nucl. Instrum. Methods A419 (1998) 326-330.

[3] M. Deile, J. Dubbert, N.P. Hessey et al., Test Beam Studies of Gas Mixtures ..., CERN ATLAS note MUON-NO-122 (1996).

[4] Common base preamplifier IO354-2 and shaping amplifier I0532-01, Instrumentation Division, Brookhaven National Laboratory, Upton, New York, 1973.

[5] C. Blocker, J. Huth, J. Oliver, Noise Considerations for the ATLAS Muon Front-End Electronics, CERN ATLAS note MUON-NO-080 (1995). 
[6] J. Huth, A. Liu, J. Oliver, Note on Noise Contribution of the Termination Resistor in the MDTs, CERN ATLAS note MUON-NO-127 (1996).

[7] Rob Veenhof, GARFIELD, a drift chamber simulation program, Version 5.35, CERN.

[8] S. Biagi, MAGBOLTZ, program to compute gas transport parameters, Version 1.10, CERN.

[9] Igor Smirnov, HEED, program to compute energy loss of fast particles in gases, Version 1.01, CERN.

[10] Landolt-Boernstein, vol. 4/3, Eigenschaften des Plasmas, no 44315, 6th edition (Springer, Berlin, Heidelberg, 1957). 